\title{
Polarimetry of near-infrared flares from Sagittarius $A^{*}$
}

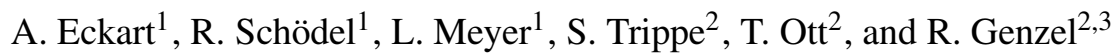 \\ 1 I.Physikalisches Institut, Universität zu Köln, Zülpicher Str. 77, 50937 Köln, Germany \\ e-mail: eckart@ph1.uni-koeln.de \\ 2 Max Planck Institut für extraterrestrische Physik, Giessenbachstraße, 85748 Garching, Germany \\ 3 Physics Department, University of California at Berkeley, Le Conte Hall, Berkeley, CA 94720, USA
}

Received 1 February 2006 / Accepted 31 March 2006

\section{ABSTRACT}

\begin{abstract}
Context. We report new polarization measurements of the variable near-infrared emission of the SgrA* counterpart associated with the massive 3-4 × $10^{6} M_{\odot}$ Black Hole at the Galactic Center.

Aims. We investigate the physical processes responsible for the variable emission from SgrA*.

Methods. The observations have been carried out using the NACO adaptive optics (AO) instrument at the European Southern Observatory's Very Large Telescope ${ }^{\star}$.

Results. We find that the variable NIR emission of SgrA* is highly polarized and consists of a contribution of a non- or weakly polarized main flare with highly polarized sub-flares. The flare activity shows a possible quasi-periodicity of $20 \pm 3$ min consistent with previous observations.

Conclusions. The highly variable and polarized emission supports that the NIR emission is non-thermal. The observations can be interpreted in a jet or temporary disk model. In the disk model the quasi-periodic flux density variations can be explained by spots on relativistic orbits around the central MBH. Alternative explanations for the high central mass concentration involving boson or fermion balls are increasingly unlikely.
\end{abstract}

Key words. black hole physics - infrared: general - accretion, accretion disks - Galaxy: center - Galaxy: nucleus

\section{Introduction}

Stellar dynamics has revealed the presence of a $(3.6 \pm$ $0.3) \times 10^{6} M_{\odot}$ Massive Black Hole $(\mathrm{MBH})$ at the center of the Milky Way at the position of the compact radio source SgrA* (Eisenhauer et al. 2005; Ghez et al. 2005; Eisenhauer et al. 2003; Schödel et al. 2003; Eckart et al. 2002; Schödel et al. 2002, for a recent review see Eckart et al. 2005). The distance to this closest $\mathrm{MBH}$ is only $7.6 \pm 0.3 \mathrm{kpc}$ (Eisenhauer et al. 2005). The close temporal correlation between rapid variability of the nearinfrared (NIR) and X-ray emission (Eckart et al. 2004; Belanger et al. 2005; Eckart et al. 2006, and Yusef-Zadeh et al. 2006) suggests that the emission with $10^{33-34} \mathrm{erg} / \mathrm{s}$ flares arises from a compact source within a few ten Schwarzschild radii $\left(R_{\mathrm{S}}\right)$ of the MBH (Baganoff et al. 2001; Baganoff et al. 2003; Eckart et al. 2004; Genzel et al. 2003a; Ghez et al. 2004; Eisenhauer et al. 2005 , and references therein). This points to a common physical origin of the phenomena (Eckart et al. 2004, 2006) and may be linked to the variability at radio through sub-millimeter wavelengths (Herrnstein et al. 2004; Mauerhan et al. 2005, and references therein).

The polarization properties of $\mathrm{SgrA}^{*}$ in the radio to sub$\mathrm{mm}$ wavelength regime are quite complex. Between 1.4 and $112 \mathrm{GHz}$ there is no significant linear polarization, with upper limits of $0.1 \%-2 \%$ (Bower et al. 1999a-c, 2001) including a detection of weak $(\sim 0.4 \%)$ circular polarization (Bower et al. 1999b). At higher frequencies of 150 to $400 \mathrm{GHz}$ Aitken et al. (2000) reported the first detection of about $10 \%$ of linear

^ Based on observations at the Very Large Telescope (VLT) of the European Southern Observatory (ESO) on Paranal in Chile; Program: 271.B-5019(A). polarization from Sgr A* (see also Bower et al. 2003; Melia \& Falcke 2001; Bower et al. 1999a; Marrone et al. 2006). From the lack of polarization at shorter frequencies, the authors concluded that the polarized flux density arises in a compact millimeter/sub-millimeter component of Sgr A*.

NIR/X-ray observations combined with theory indicate that the $\mathrm{MBH}$ source $\mathrm{SgrA} *$ is strongly variable with a NIR/X-ray spectrum involving Synchrotron self-Compton models (Eckart et al. 2004, 2006) and rapid cooling of transiently heated electrons, Doppler beaming, and modulation due to special relativistic aberrations associated with the relativistic orbital motion (Broderick \& Loeb 2006; Eisenhauer et al. 2005; Ghez et al. 2004; Gillessen 2006) or a short jet (Melia \& Falcke 2001; Markoff et al. 2001; Falcke et al. 2000; Yuan et al. 2002). Models show that polarized emission may arise in ordered toroidal magnetic fields of a disk-like accretion flow, where the $\boldsymbol{E}$-vector of the $20 \%$ to $60 \%$ polarized radiation may be perpendicular to the equatorial plane (Broderick \& Loeb 2006; Falcke et al. 2000; Goldston et al. 2005). However, magnetic field configurations in accretion flows and (temporal) disks may have a strong turbulent component as well (Shakura \& Sunyaev 1973; Duschl et al. 1991; Balbus 2003). At NIR wavelengths depolarization due to the Faraday effect or Thompson scattering can be neglected. The NIR emission can then be expected to be significantly polarized, as already detected previously in a single weak flare (Genzel et al. 2003a).

Here we report $\sim 3$ min time resolution NIR polarization measurements of a flare from the infrared counterpart of SgrA* which clearly shows that the emission is non-thermal. These data are of high interest because the polarized light in the NIR regime may carry information on the relativistic plasma close to the event horizon. During a >100 min flare in July 2005 we 
detected 3 short sub-flares with a polarization of up to $25 \%$ and an associated swing in polarization angle of about $\sim 40^{\circ}$ following the peak total and polarized intensities.

In Sect. 2 we describe the observations and data reduction and present the polarized NIR flux density light curves and polarization properties of SgrA* as a function of time in Sect. 3. The data is interpreted in the light of a possible jet and an orbiting spot model in Sects. 4 to 6. A summary and conclusions are given in the final Sect. 9. A characterization of the observed variability as well as alternative models to the $\mathrm{MBH}$ hole scenario are discussed in the appendix.

\section{Observations and data reduction}

The observations of SgrA* have been carried out in the NIR $K_{\mathrm{S}}$-band $(2.0-2.36 \mu \mathrm{m})$ using the NIR camera CONICA and the adaptive optics (AO) module NAOS on the European Southern Observatory's Very Large Telescope $\mathrm{UT}^{1}{ }^{1}$ on Paranal, Chile, during the nights between 12 and 13 June 2004 and 29 and 30 July 2005. The infrared wavefront sensor of NAOS was used to lock the AO loop on the NIR bright ( $K$-band magnitude (6.5) supergiant IRS 7, located about 5.6" north of Sgr A*. On both occasions the atmospheric conditions (and consequently the AO correction) were stable during the observations with optical seeing values ranging between $0.5^{\prime \prime}$ and $0.8^{\prime \prime}$. Therefore the AO could provide a stable correction with a high Strehl ratio (of the order $50 \%$ ).

In NACOS/CONICA (NACO) the combination of a Wollaston prism with a half-wave retarder plate allows the simultaneous measurement of two orthogonal directions of the electric field vector and the rapid change between different angles, both crucial when trying to determine the polarization characteristics of a time varying source. In July 2005 six and June 2004 four polarization angles could thus be observed in rapid sequence within less than $3.5 \mathrm{~min}$ which is short compared to the typical time scales expected for flares (Eckart et al. 2004; Genzel et al. 2003a; Ghez et al. 2004; Eckart et al. 2006). Observations of a dark cloud a few arc-minutes to the north-west of Sgr A* were interspersed with exposures of the target in order to obtain sky measurements. All observations were dithered (in order to cover a larger area and improve the image quality), sky subtracted, flat-fielded, and corrected for bad pixels. PSFs (point spread functions) were extracted from all the single exposures using the StarFinder code (Diolaiti et al. 2000). The individual exposures were deconvolved with the Lucy-Richardson and with a Wiener filter algorithm and beam restored with a Gaussian beam of FWHM corresponding to the respective wavelength (60 milli-arcseconds at $2.2 \mu \mathrm{m})$. Flux densities of compact sources were obtained via aperture photometry on the diffraction limited images. These values were corrected for a background flux density contribution. This background was determined as the mean value of fluxes measured in the same size apertures at 5 different positions in a field $\sim 0.5^{\prime \prime}$ to $1.5^{\prime \prime} \mathrm{NW}$ of SgrA* which is free of obvious contributions from individual stars. Estimates of uncertainties were obtained from a comparison of these results and from the standard deviation of fluxes of nearby constant sources of similar brightness as $\operatorname{Sgr}{ }^{*}$. The calibration was performed using polarization measurements of IRS21 $(\sim 13 \pm 4 \%$ at PA $\left.\sim 14^{\circ}\right)$ and the overall interstellar polarization of all sources in the field of 3-4\% at $\sim 25^{\circ}$ (Knacke \& Capps 1977; Lebofsky et al. 1982; Ott et al. 1999; Eckart et al. 1995).

\footnotetext{
${ }^{1}$ Based on observations at the Very Large Telescope (VLT) of the European Southern Observatory (ESO) on Paranal in Chile; Program: 075.B-0093.
}

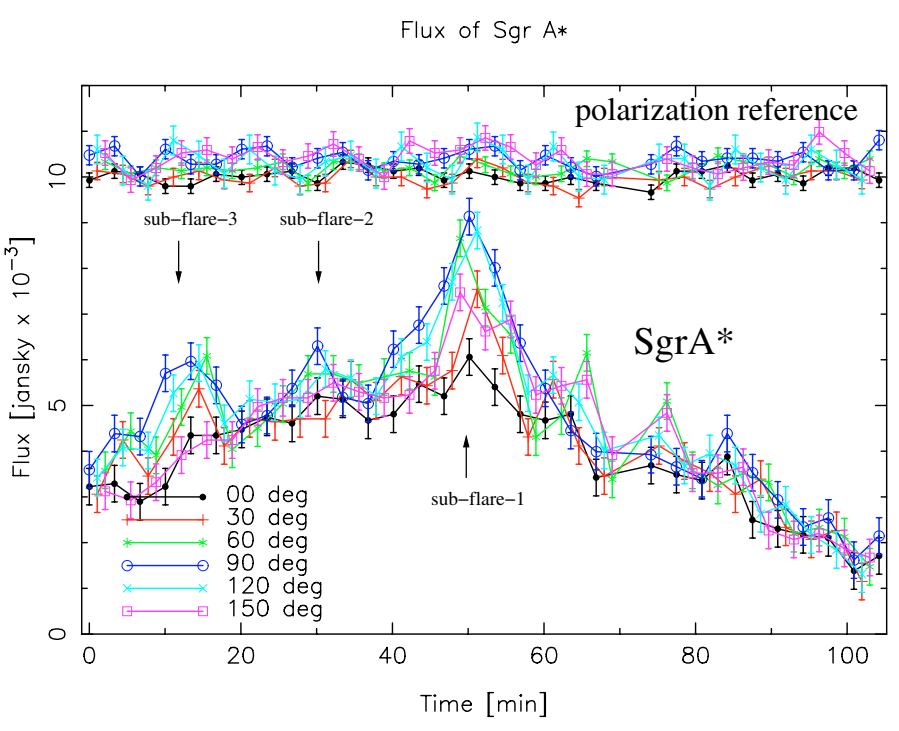

Fig. 1. Flux density at different PAs of the Wollaston prism for the polarization reference star $\sim 0.38^{\prime \prime}$ north of SgrA* (Fig. 2) and the NIR counterpart of SgrA* on the nights between 29 and 30 July 2005. Six polarization angles $0^{\circ}, 30^{\circ}, 60^{\circ}, 90^{\circ}, 120^{\circ}$ and $150^{\circ}$ (East of North) were used. For display purposes the flux density values of the reference star have been shifted upwards by $5 \mathrm{mJy}$.

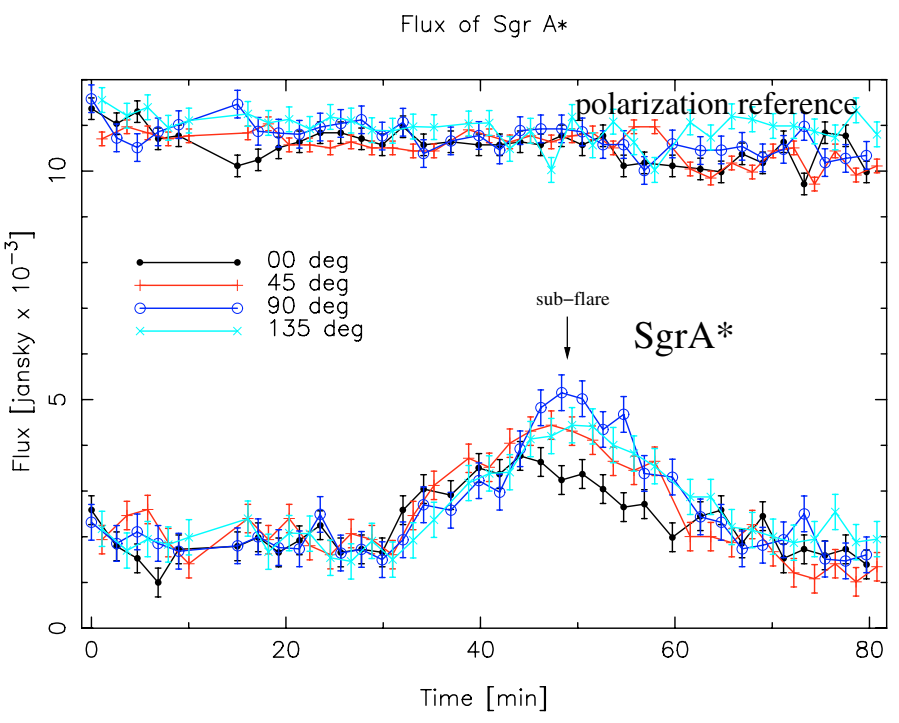

Fig. 2. Flux density at different PAs of the Wollaston prism for a polarization reference star near SgrA* and the NIR counterpart of SgrA* taken on the nights between 12 and 13 June 2004. Four polarization angles $0^{\circ}, 45^{\circ}, 90^{\circ}$, and $135^{\circ}$ (East of North) were used. For display purposes the flux density values of the reference star have been shifted upwards by $5 \mathrm{mJy}$.

\section{Polarized NIR light curves from SgrA*}

In Figs. 1 and 2 we show the flux density at different position angles (PA) of the Wollaston prism for a polarization reference star and the NIR counterpart of SgrA* in July 2005 and June 2004 with 6 and 4 polarization angles, respectively. These data clearly show that the polarization is significant in certain parts of the overall light curve. Since the overall light curve is termed "flare", we give the shorter polarized events the term "sub-flare" in the following. Figure 3 (top right) shows the mean of our $K$-band NACO images of the central $0.6 \times 0.6 \operatorname{arcsec}^{2}$ and at $0^{\circ}$ and $90^{\circ}$ polarization angle taken simultaneously through the Wollaston 

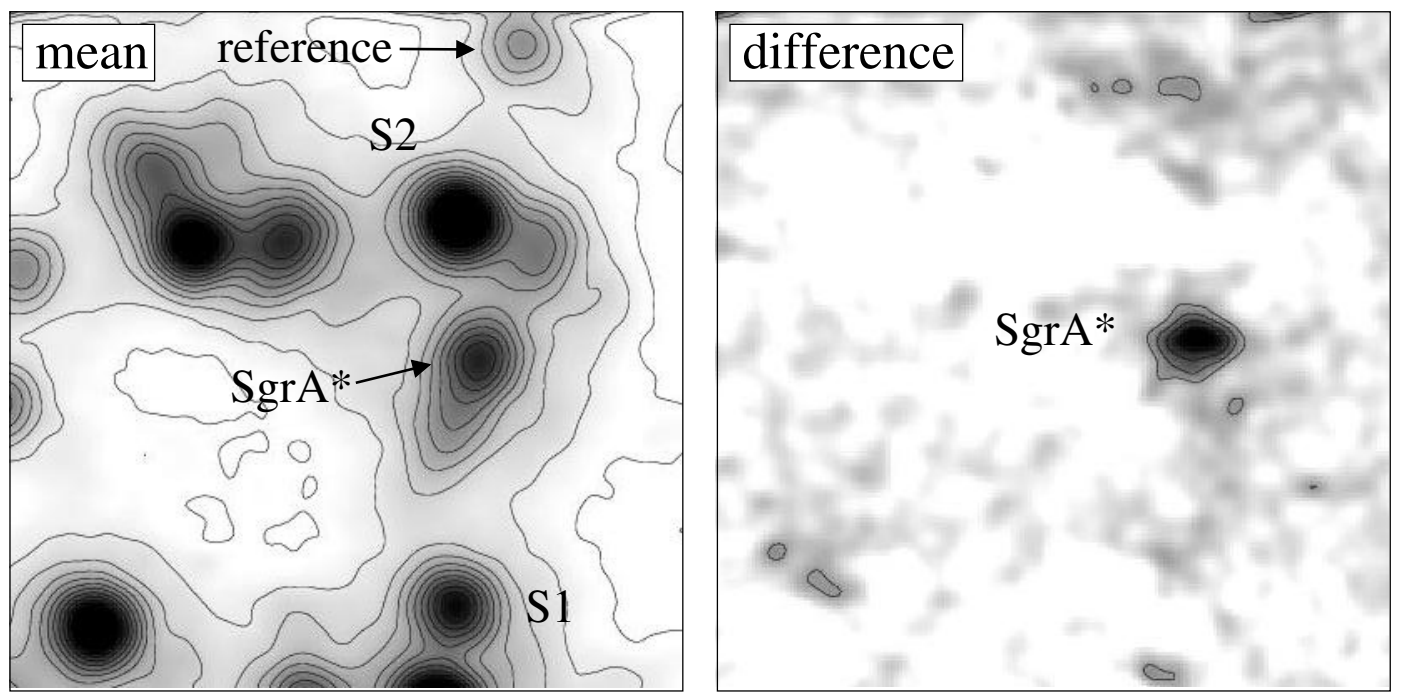

Fig. 3. The mean (left) and difference (right) of our $K$-band NACO images at $0^{\circ}$ and $90^{\circ}$ polarization angle taken simultaneously through the Wollaston prism during the peak of the flare at $t=50$ after the start of the measurements in July 2005 (see Fig. 1).
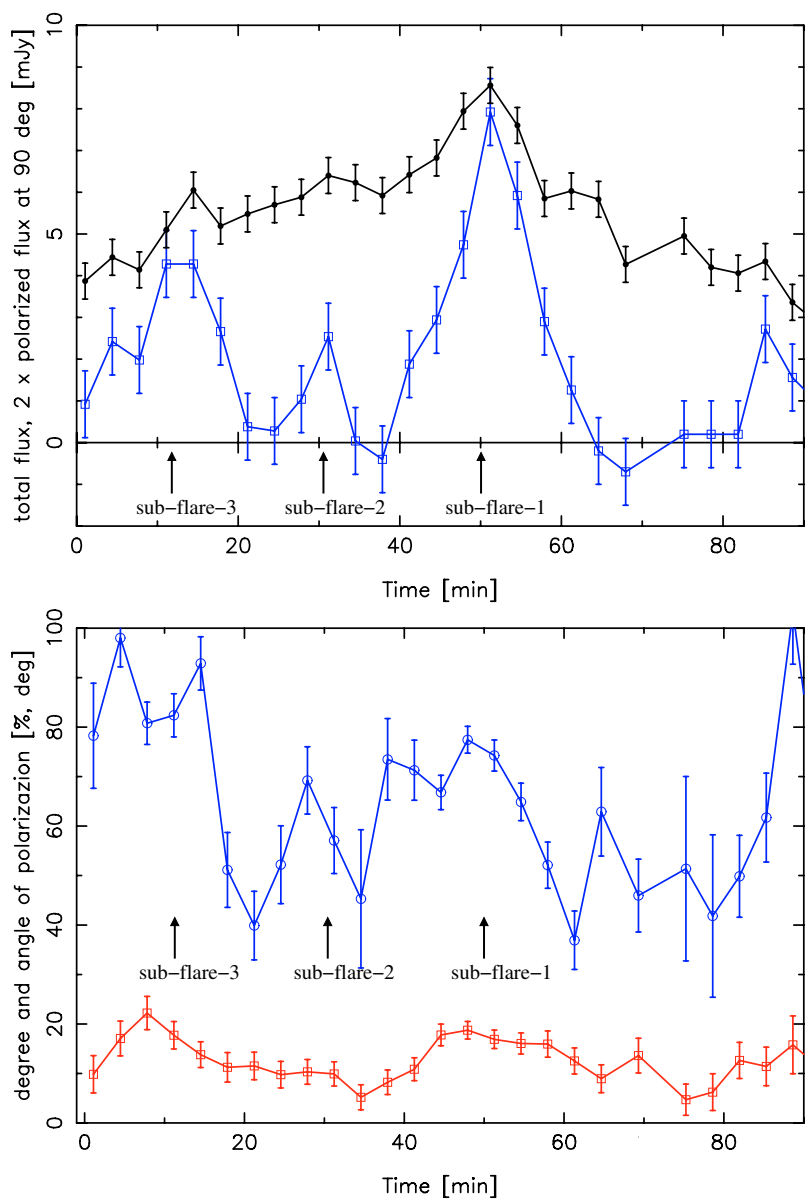

Fig. 4. Polarized emission from the NIR counterpart SgrA* in July 2005. Top: the total de-reddened flux density light curve (black) and the de-reddened flux density at polarization angle $90^{\circ}$ (East over North; blue) corrected for the flux density measured at a PA of $0^{\circ}$ at which the sub-flares cannot be seen. Bottom: the position angle of the $\boldsymbol{E}$-vector (top graph, blue) and the degree of polarization (bottom graph, red). The left axis is used to label both quantities in different units as given in brackets. The arrows are separated by $20 \mathrm{~min}$ (see Fig. 6) and mark the position of the three sub-flares. Another weak peak in polarized flux that is in agreement with the observed quasi-periodicity can be seen at $t=85$. prism during the peak of the flare at $t=50$ as plotted in Fig. 1 . The polarization reference star (Fig. 3) is labeled as "reference". The bottom right panel in Fig. 3 shows the difference between these $90^{\circ}$ and $0^{\circ}$ images clearly showing the excess of polarized de-reddened flux at the position of SgrA* for the strongest sub-flare-1 in Fig. 1. The top panels of Figs. 4 and 5 show the total de-reddened flux density light curve and the de-reddened flux density at polarization angle $90^{\circ}$ as a function of time. The bottom panels of these figures show the position angle of the $\boldsymbol{E}$-vector and the degree of polarization.

In July 2005 (Fig. 4) the polarized sub-flares with FWHM durations of the order $10 \mathrm{~min}$ are superimposed on top of the broader main flare. The three sub-flares show peak polarizations of $12-25 \%$ with a swing in polarization angle of up to $40^{\circ}$. The most convincing swing occurs between $t=50$ and $t=60$ in the July 2005 data. At $t=35$ and $t=75$ the polarization is close to the galactic background polarization level of $\sim 3 \%$ at $\sim 25^{\circ}$ measured for the stars. The most obvious sub-flares are those labeled sub-flares- 1 and sub-flares- 3 in Fig. 4. Sub-flares- 2 is weaker. It is a $2-3 \sigma$ event at position angles of $60^{\circ}$ and $90^{\circ}$ and a $1 \sigma$ event at a PA of $0^{\circ}$. At $t=35-40$ sub-flares- 2 is followed by a local minimum at all PAs except $30^{\circ}$. We therefore consider it as a significant sub-flare.

Marginal flux density excursions on the $1-2 \sigma$ level occur at $t=65,75$ and 85 . The 2-3 $\sigma$ flux density excursions at $t \sim 65$ and $t \sim 75$ only occur at the orthogonal PAs of $60^{\circ}$ and $150^{\circ}$. They therefore result from a single Wollaston prism setting and are most likely due to a residual uncertainty in the flux density calibration of the individual channels. We consider these excursions as not significant.

In general the polarized sub-flares are not clearly visible at polarization channels around $\sim 0^{\circ}$ position angle which shows the least polarized flux density. This indicates that the underlying broader flare is not significantly polarized (Figs. 1 and 4) and that the sub-flares may be intrinsically highly polarized - possibly up to $60 \%$ (Broderick \& Loeb 2006; Falcke et al. 2000; Goldston et al. 2005).

In June 2004 (Figs. 2 and 5) a single polarized sub-flare with FWHM duration of the order $10 \mathrm{~min}$ is superimposed on top of the broader main flare with a FWHM duration of about 20 min. The first section of the overall flare $(t=30-45)$ indicates that the broader underlying flare shows little $(\leq 10 \%)$ or no polarization. 

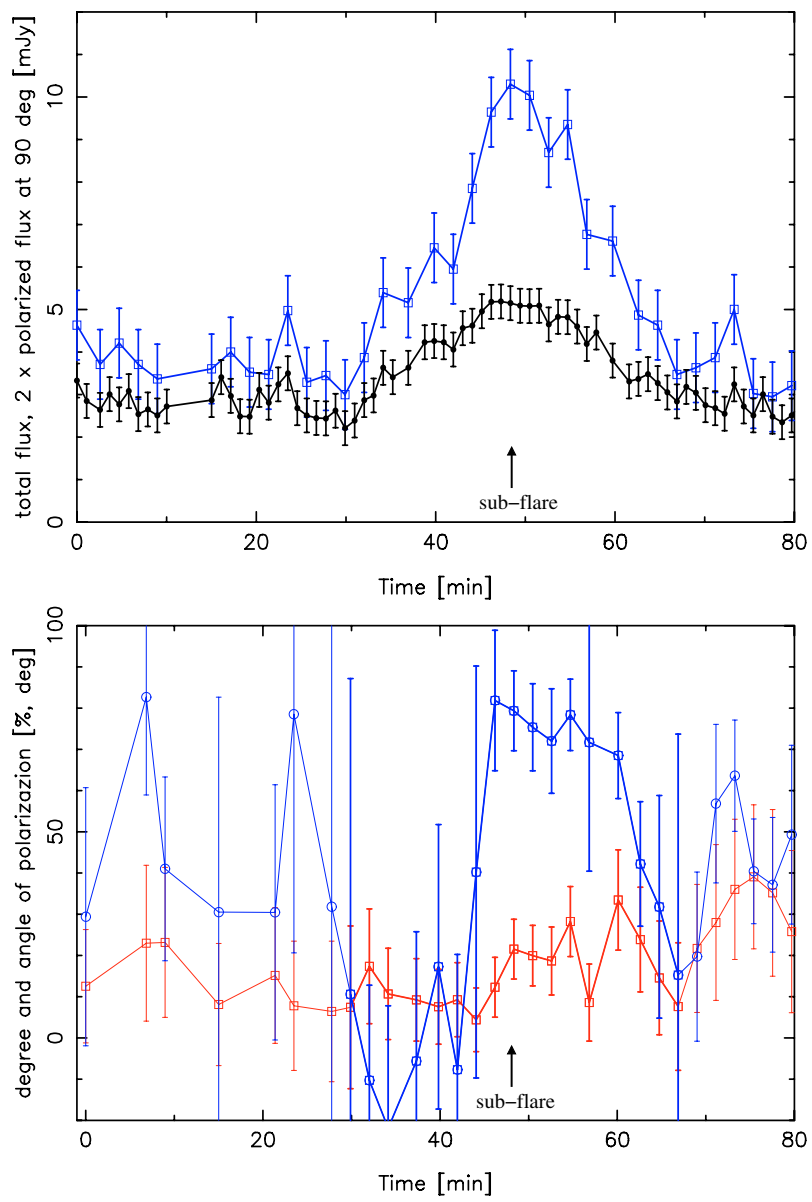

Fig. 5. Polarized emission from the NIR counterpart SgrA* in June 2004. Top: the total de-reddened flux density light curve (black) and the de-reddened flux density at polarization angle $90^{\circ}$ (East over North; blue) corrected for the flux density measured at a PA of $0^{\circ}$ at which the sub-flares cannot be seen. Bottom: the position angle of the $\boldsymbol{E}$-vector (top graph, blue) and the degree of polarization (bottom graph, red). The left axis is used to label both quantities in different units as given in brackets. The arrow marks the position of the polarized sub-flare. The region over which the flare emission is strong have been highlighted by bold face lines. Over the remaining sections of the plot the flare emission is weak and the error bars are correspondingly large. For completeness, however, we have included these data points as well.

Its start preceeds the start of the significantly polarized sub-flare at $t \sim 45$ by about $10-15 \mathrm{~min}$. This is best seen in Fig. 2. In Fig. 5 (top) we compare the polarized flux density at a PA of $90^{\circ}$ to the total flux density of the flare. In this figure the fact that the polarized sub-flare starts after the overall flare emission is less obvious since the total flux density contains both the polarized and unpolarized flux density. With about $20 \%$ the overall degree of polarization and a swing in position angle of about $40^{\circ}-50^{\circ}$ the properties of the June 2004 sub-flare are very much comparable to the sub-flares observed in July 2005.

Theoretical calculations indicate that the observed polarization properties of SgrA* may trace the relativistic accretion flow in a strong gravitational field and that the polarization can be used to probe the spacetime at the photon orbit (Falcke et al. 2000; Broderick \& Loeb 2006; Goldston et al. 2005).

\section{The jet model}

Although in the case of SgrA* no jet has been positively detected yet, our new NIR data could be taken as an indication for a relativistic jet structure. The rotation of the polarization angle could be due to a helical magnetic field along the jet and the variable sub-flare emission could be explained by temporal instabilities in the jet.

In compact extragalactic radio sources the distribution between the $\boldsymbol{E}$-vector position angles of the cores or jet components and the jet axis show a rather flat distribution with the tendency of perpendicular orientations for QSOs (Pollack et al. 2003) and a weak indication for stronger beamed sources to give a parallel arrangement (Rusk 1988; Gabuzda et al. 2000; but see also Pollack et al. 2003).

This can be understood in the framework of polarized radiation from oblique and conical shocks (Cawthorne \& Cobb 1990) and sources seen at inclinations where the $\boldsymbol{E}$-vector is parallel to the jet axis will undergo the strongest variability due to relativistic beaming. This is consistent with the finding of Gomez et al. (1994) who show that in helical jets, the strongest emission is obtained when the shock wave reaches regions where the jet is bent towards the observer. There the maximum Doppler boosting, the lengthening of the integration column, and the shock effects simultaneously contribute to the emission. At these locations the electric vector lies parallel to the jet axis, due to the enhancement of the magnetic field parallel to the shock front. However, multi frequency observations of nuclear jets at $\mathrm{cm}$ wavelengths also indicate polarization structure transverse to the jet axis (Pushkarev et al. 2005). Along the bright spine of the jet the polarization $\boldsymbol{E}$-vectors are aligned with the jet. Within the "sheaths" at both edges of the jet the $\boldsymbol{E}$-vectors are orthogonal to the jet axis. The authors identify the presence of polarization aligned with the jet near the "spine" as an indication for the presence of a helical B-field that propagates outward with the jet flow.

Polarized radio radiation and swings in position angles of several $10^{\circ}$ have also been observed for micro-quasars (Fender et al. 2000, 2003) and low luminosity AGN (e.g. Bower et al. 2002a,b). The comparison to low luminosity galactic and luminous extragalactic sources shows that a jet or outflow phenomenon possibly provides an explanation of our NIR data and that the $\boldsymbol{E}$-vector may lie along the jet axis (or perpendicular to it). A longer jet may have the potential of showing sub-flares that would stay bright for a time exceeding the 10 min reported here. Such a jet could be responsible especially for the emission at FIR/mm-wavelengths (Yuan et al. 2002).

There is not firm observational evidence for a jet or collimated outflow from SgrA* (see, however, discussion in Sect. 6). Therefore we have concentrated our interpretation on a disk model in which the flare activity is due to luminous spots that are on relativistic orbits around the central MBH. In fact, near the last stable orbit (LSO) a short jet emerging from a disk may likely look almost indistinguishable from a case involving a pure disk or orbiting spots. This fact is supported by recent VLBI measurements of the intrinsic size of SgrA* at mm-wavelengths (see Fig. 4 in Bower et al. 2004). In addition the emission at the even shorter NIR wavelengths are likely to come from even smaller scales in the jet model.

\section{The orbiting spot model}

Our polarization measurements are consistent with emission from highly polarized bright spots in relativistic orbits around the MBH (e.g. Dovciak et al. 2004; Broderick \& Loeb 2006; Goldston et al. 2005). We find that the sub-flares have a duration that is consistent with the flux density amplification peaks expected for spots orbiting near the last stable orbit (LSO). 

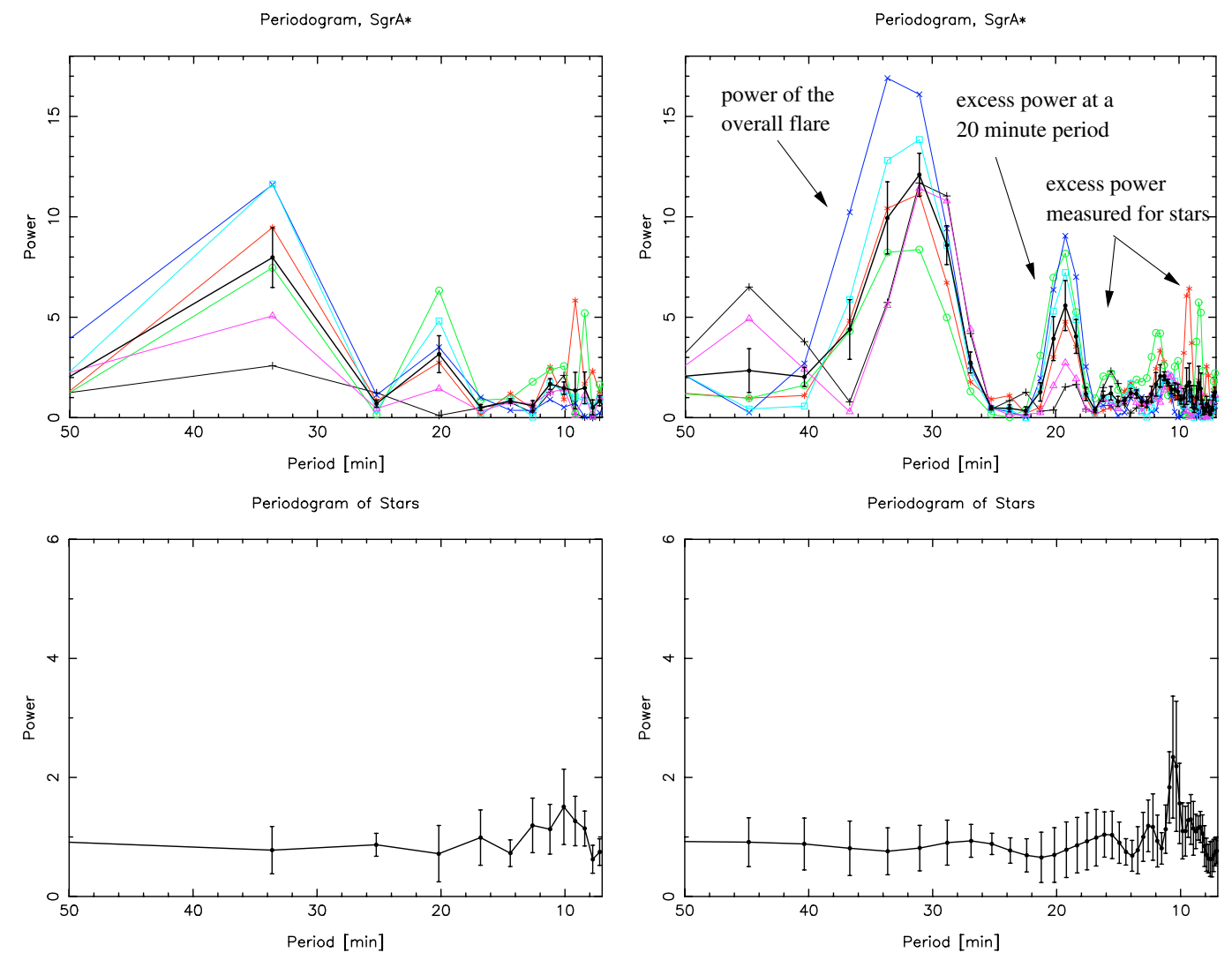

Fig. 6. Top left: periodogram of the July 2005 polarized flux density data from SgrA* at individual polarization angles (see color coding information in Fig. 1) and for the mean (black). Bottom left: mean and standard deviation calculated from periodograms of 7 stars within 1.5 arcsec radius of SgrA*. Top and Bottom right: the same as top and bottom right but oversampled in order to allow a better estimate of the peak widths. Note that both the mean and PA $0^{\circ}$ curves are printed in black. They can, however, be clearly distinguished from each other since only the data points of mean curve have error bars associated with them.

Therefore the sub-flares may arise from spots with a size of $\lesssim R_{\mathrm{S}}$ embedded in a low polarization temporarily present (or bright) disk or ring at a distance of a few $R_{\mathrm{S}}$ possibly close to the LSO. In such a two component model the disk would be responsible for the not significantly polarized overall flare and dilute the polarization signal from the higher polarized spots. The time difference between the observed bright polarized sub-flares is about $20 \mathrm{~min}$ - similar to the quasi-periodicity oscillations (QPO) reported previously (Genzel et al. 2003a). A total flux density periodogram of the July 2005 data shows power at a period of $20 \pm 3$ min (Fig. 6). Given that from our data there is no evidence for red-noise excess power at periods well above $20 \mathrm{~min}$ beyond the $1-2 \sigma$ level (see discussion on periodograms and red-noise in Appendix A) we regard the power at a period of $20 \pm 3 \mathrm{~min}$ as significant. The stars show no excess power at a period of $20 \mathrm{~min}$. The top and bottom left panels in Fig. 6 show the results of the analysis oversampled in order to allow a better estimate of the peak widths.

It is conceivable that the 3 sub-flare events observed in July 2005 are associated with the same spot. The required lifetime of a few $10 \mathrm{~min}$ to half an hour can well be matched by the expected synchrotron cooling time scale of a flare given via $t_{\mathrm{s}} \sim 5 \times 10^{8} v_{9}^{-0.5} B^{-3 / 2}$, where $t_{\mathrm{s}}$ is in seconds, $B$ is in Gauss, $v_{9}$ is the self-absorption cutoff frequency of the synchrotron spectrum in $\mathrm{GHz}$. For THz peaked spectra and typical magnetic fields of several $10 \mathrm{G}$ (Eckart et al. 2004, 2006) the synchrotron cooling time at the peak is of the order of $1 \mathrm{~h}$ and represents an upper limit for the duration of the acceleration process that may also provide electrons radiating in the NIR. In the NIR/MIR domain at frequencies around $100 \mathrm{THz}$ the cooling time scale would be of the order of the observed polarized sub-flare time scale. Under these circumstances an orbiting spot could stay bright for a few orbital time scales (see Eckart et al. 2006; Gillessen 2006). The properties of the flare emission observed in June 2004 are consistent with the above interpretation. The only difference is that the overall flare event was about 5 times shorter and only one polarized sub-flare was observed.

\section{On possible evidence for collimated outflows}

The mean position angle of the $\boldsymbol{E}$-vector may reveal information on the orientation of the spin axis of the temporary disk or MBH or even a possible jet or outflow. Here we summarize the possible evidence that can be found for the jet and orbiting disk scenario.

\subsection{Evidence for a jet?}

In the case of $\mathrm{SgrA}^{*}$ there is no direct observational evidence for a jet at radio and mm-wavelengths. However, the jet model is highly successful in explaining the radio observations (e.g. Bower et al. 2004). There are, however, two conspicuous features that may be taken as an indication for a highly collimated outflow approximately perpendicular to the position angle of the $\boldsymbol{E}$-vector. Morris et al. (2004) report on a thin elongated feature, a "streak", in the CHANDRA X-ray image. We labeled this feature XF in the inset of Fig. 9. This feature has a minimum length of $9^{\prime \prime}(0.23 \mathrm{pc})$, is located at a mean distance of $\leq 16.8^{\prime \prime}$ $(\leq 0.66 \mathrm{pc})$ to the SE (not SW as quoted by Morris et al. 2004) 
from the center and points at a position angle of $\sim 30^{\circ}$ to within $\leq 5^{\circ}$ into the direction of SgrA*.

In the thermal IR domain we find a comparable structure. In addition to a few sharp edged structures associated with the northern arm of the mini-spiral (e.g. Clénet et al. 2004) one can see an almost linear feature (LF) extending over 2 thirds of an arcsecond and pointing at a position angle of $\sim 60^{\circ}$ to within $\leq 10^{\circ}$ into the direction of SgrA* (see Fig. 9). The NIR filament LF and the X-ray filament XF are not colinear.

The feature LF and an extended structure labeled EF in Figs. 8 and 9 are of special interest, as their geometry with respect to SgrA* rises the question if they could be outflow signatures. LF indeed qualitatively shows similarity to a jet; but the fact that it is bent and not pointing directly towards SgrA* may question this impression. The EF is known as the "Finger" (Vollmer \& Duschl 2000) or "Tip" of the Eastern arm to which it connects through a bubble-like feature called the "microcavity" (Paumard et al. 2004). The fact that EF is more difficult to identify in Fig. 8 compared to Fig. 9 indicates that there is no filamentary structure contained in EF that is as sharp as that of feature LF. The dynamics of this feature were examined by Paumard et al. (2004), who constructed velocity maps based on $\mathrm{Br} \gamma$ line maps obtained with integral field spectroscopy. Those velocity maps show a high radial velocity $\left(\sim 300 \mathrm{~km} \mathrm{~s}^{-1}\right)$ of the Tip and a very steep velocity gradient $\left(\sim 80 \mathrm{~km} \mathrm{~s}^{-1}\right)$ within this structure. This clearly indicates, that EF is highly inclined with respect to the plane of sky, leaving two possible interpretations:

(1) the Tip is indeed an interaction zone between the Galactic Center ISM and the top of a jet from SgrA*; in this case it would be located behind the plane of the black hole;

(2) the Eastern arm is a "quiet" flow in front of the plane of SgrA* which undergoes a dramatic loss of momentum due to the interaction with a stellar wind at the location of the microcavity, and then starts falling onto SgrA* through the Tip. In that hypothesis, the microcavity could also be due to an interaction not with a stellar wind, but with a jet.

In any case the Tip itself would then be the signature of an inflow rather than an outflow. Combining the existing information, interpretation (2) appears more probable than (1) as in Bry line maps the northern edge of the Eastern arm appears more excited than the rest of it. The cause for this is either a shock with the Eastern bridge (which is definitely in front of the Northern arm, if not of $\operatorname{SgrA} *$ ), or because we mostly see the "dark side" of the Eastern arm, and this more excited edge pertains to the "bright side".

While the above described features observed in the X-ray and thermal IR domain may indicate a collimated outflow such a phenomenon requires better proof. An alternative explanation for these narrow and elongated features are that they belong to the class of non-thermal filaments (NTFs; see e.g. LaRosa et al. 2004; Nord et al. 2004; and for reviews Morris 1996; Morris \& Serabyn 1996).

\subsection{The spin of a disk or $\mathrm{MBH}$}

The mean position angle of the $\boldsymbol{E}$-vector may be linked to the orientation of the spin axis of the disk that may be temporarily present (or bright) during the flares. At small radii the flow's angular momentum may be tied to the $\mathrm{MBH}$, which it accreted over its lifetime (Broderick \& Loeb 2006). The flow orientation may also be set by the angular momentum of the stars whose winds feed the MBH (Genzel et al. 2003a; Levin \& Beloborodov 2003; see also Bardeen \& Petterson 1975). For both data sets
(July 2005 and June 2004) we observe a polarization PA that varies by $\pm 20^{\circ}$ about a mean angle of $\sim 60^{\circ}$ (E of $\mathrm{N}$ ) and does not wrap around the $0^{\circ} / 180^{\circ}$ limit. To within the uncertainties this mean angle is the same for all observed sub-flares. If we assume that the sky projected magnetic field component parallel to the inclination axis of the disk is dominant then the distribution of PAs is not wrapped and the mean PA of the $\boldsymbol{E}$-vector indicates the projected spin orientation on the sky. Such a scenario may be present over most parts of an inclined disk with a toroidal field structure or in case of a strong turbulent component with an isotropic distribution of magnetic field lines in or close to the disk plane (Shakura \& Sunyaev 1973; Duschl et al. 1991; Balbus 2003).

Figure 7 shows model calculations of the peak normalized flux density, degree of polarization, polarized flux density and PA of the $\boldsymbol{E}$-vector as calculated for orbiting spot models based on the code by Dovciak et al. (2004). The calculations were done for a single spot with a FWHM of about one Schwarzschild radius, spin parameters of $a=0.0, a=0.5, a=0.998$, orbiting at the corresponding last stable orbits. We assumed that the intrinsic $\boldsymbol{E}$-vector is perpendicular (i.e. NS at a PA of $0^{\circ}$ ) to the orbital plane. The calculations show that for inclinations $\gtrsim 50^{\circ}$ and spins below $a \lesssim 0.5$ the polarization angle does not wrap around the $0^{\circ} / 180^{\circ}$ limit but varies over the flare close to a value expected for a non-relativistic orbiting disk (see also Eq. (4.6) in Bao et al. 1997).

We find that in two independent data sets (July 2005 and June 2004) the PA does not wrap as expected for inclinations $\gtrsim 50^{\circ}$ and spin values $a \lesssim 0.5$. Under these conditions (see Fig. 7) the polarization angle varies over the flare close to a value expected for a non-relativistic orbiting disk and the mean orientations of the projected $\boldsymbol{E}$-vector agrees with that of the projected spin axis. This statement also holds for more sophisticated physical models of the intrinsic polarization (Broderick \& Loeb 2006), as long as the observed distribution of PAs is not wrapped around the $0^{\circ} / 180^{\circ}$ limit.

Based on these theoretical predictions and on our observational data we therefore speculate that the measured $\boldsymbol{E}$-vector of the polarized emission may indicate the projected spin axis of a temporary disk around $\mathrm{SgrA}^{*}$. In this picture an associated outflow would be perpendicular to the disk and therefore also perpendicular to the jet like features mentioned in Sect. 6.1. Currently it is unclear how the direction of the projected $\boldsymbol{E}$-vector can be related to the various structures observed in the central parsec. In Fig. 8 we show the range of orientations of the electric field vector on the sky. It points approximately into the direction of the mini-cavity, which has been interpreted previously as being the result an interaction of the mini-spiral with a wind out of the general direction towards SgrA* (Lutz et al. 1993; Melia et al. 1996; Yusef-Zadeh et al. 1998). The projected $\boldsymbol{E}$-vector is at large angles $\left(\gtrsim 50^{\circ}\right)$ with the spin vectors of all planar structures except the bar component and the system of clockwise orbiting young and luminous stars with high massloss (Levin \& Beloborodov 2003; Genzel et al. 2003b; Paumard et al. 2006, submitted to ApJ). These planar structures may be related to a preferred plane over which the SgrA* MBH accretes matter (Broderick \& Loeb 2006; Melia \& Falcke 2001; Genzel et al. 2003b; Levin \& Beloborodov 2003).

\section{A two component hot spot/disk model}

In order to demonstrate the consistency between the hot spot model with the observations we present a possible solution for the July 2005 measurements (a similar model can be obtained 

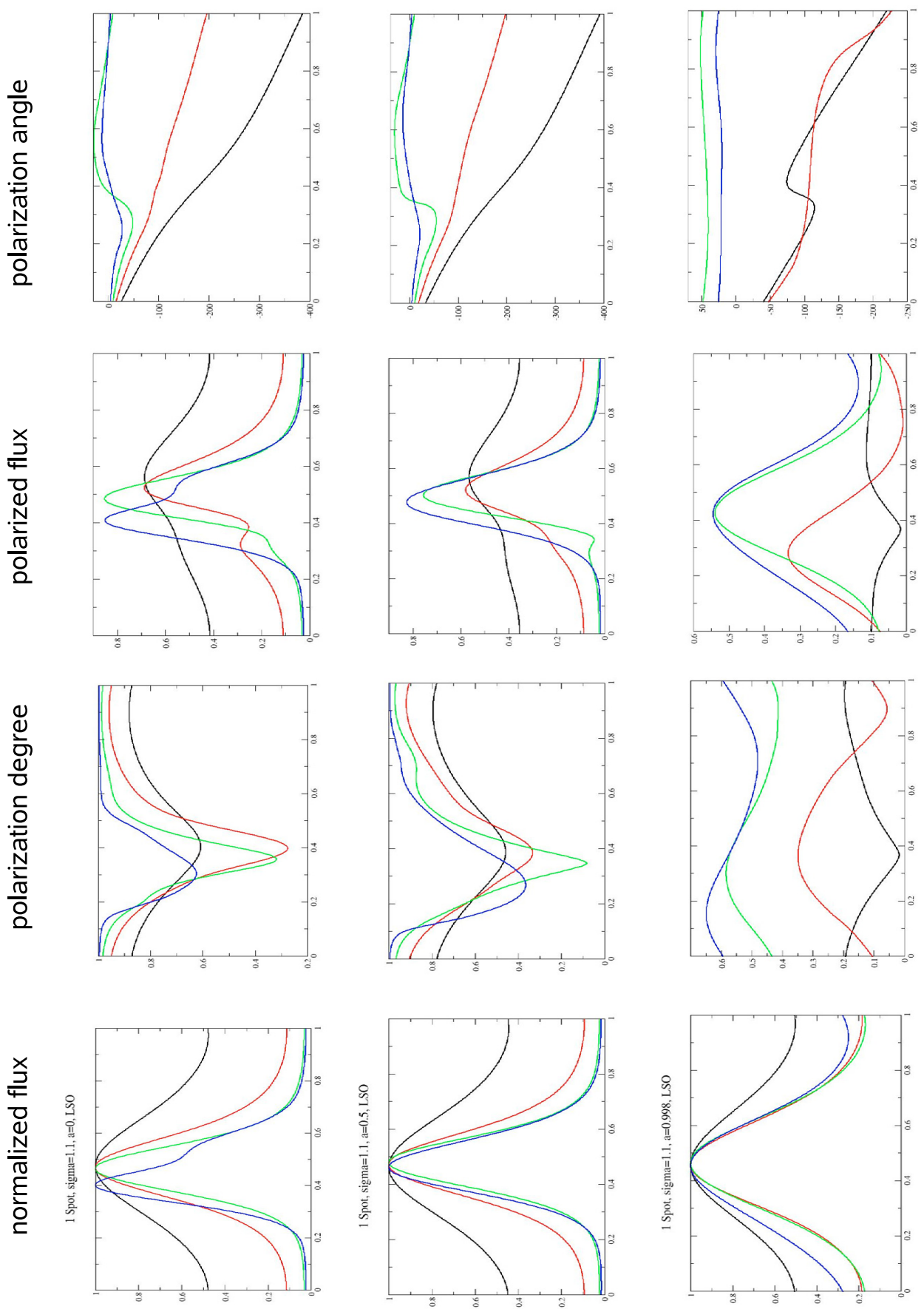

Fig. 7. Curves of peak normalized flux density, degree of polarization, polarized flux density and PA of the $\boldsymbol{E}$-vector as calculated for orbiting spot models based on the code by Dovciak et al. (2004). The data are shown for one orbital period. The inclinations are color coded as $10^{\circ}$ black, $30^{\circ}$ violet, $50^{\circ}$ green, $70^{\circ}$ blue.

for the June 2004 data). The QPOs find a natural explanation in a compact emission region that orbits around the MBH. In addition to the hot spot picture required to model the sub-flares, we use a time varying underlying disk that generates the overall flare. For the demonstration purposes we have chosen to match the observed data on a visual basis. In what follows we describe how the modeling (see Fig. 2) was done and try to give constraints on the used parameters.

The calculations have been carried out using the code by Dovciak et al. (2004). An optically thick, geometrically thin emission region that lies within the equatorial plane has been assumed. For the intrinsic emissivity of the disk we chose a
Gaussian shaped time dependency and a $r^{-1}$ spatial dependency (following Yuan et al. 2004). We assumed a polarization degree of 3\%. Higher intrinsic polarization would not allow for the observed changes in the polarization angle. The spot in our case has a radial extent of one $R_{\mathrm{S}}$ and an orbital Gaussian shaped extent of $\sigma \sim 1.5 R_{\mathrm{S}}$. The size was chosen in such a way that on the one hand the peaks in the flux curve are matched well and that on the other hand the changes in the polarization angle are not too small. The center of the spot is roughly located on the LSO so that for a MBH with $a=0.5$ the orbital period of $\sim 20 \mathrm{~min}$ is matched. We have not considered shearing of the spot and assume that it remains confined over a few 


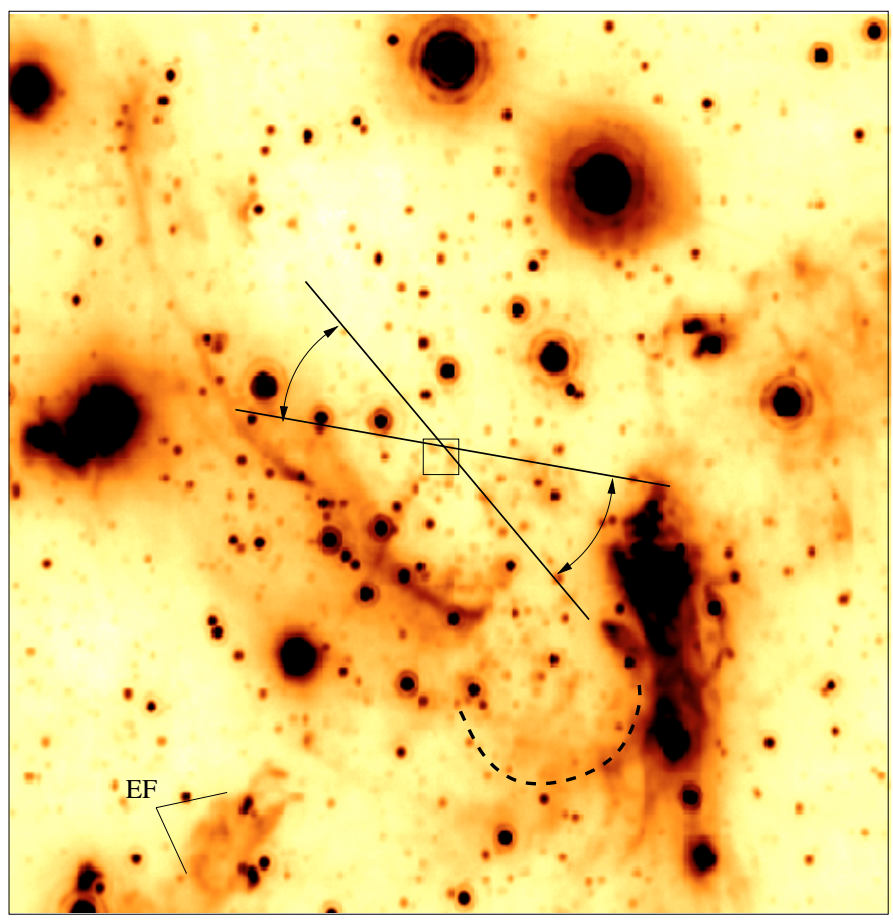

Fig. 8. An image of the central $0.5 \times 0.5$ square parsec $(1$ arcsec $\sim 0.039$ parsecs) of the Galactic Center at a wavelength of $3.8 \mu \mathrm{m}$ using the NACO adaptive optics system at the VLT. In addition to the stars the dust emission from the min-spiral is seen. The rectangular box indicates the image section shown in Fig. 3. The two lines centered on the position of SgrA* cover the range over which the polarization angle observed in July 2005 and June 2004 varies on the sky. The approximate location and shape of the mini-cavity is shown as a dashed line. The source EF is described in the text and marked in Fig. 9.

orbits. In order to match the variable observed flux density of the sub-flares, we changed the intrinsic luminosity for every period. Normalized to sub-flare-1 (Fig. 4), the spot has 50\% intensity during the first period (corresponding to sub-flare-3), $20 \%$ in the second (corresponding to sub-flare-2), and $10 \%$ in the fourth (corresponding to $t \sim 64$ ). During the third revolution, the spot has a peak over-brightness relative to the disk (at the spot's location) of roughly $50 \%$ at the chosen inclination of $55^{\circ}$. We used this inclination, because in our model the polarization angle does not wrap around the $0^{\circ} / 180^{\circ}$ limit only for viewing angles $\geq 50^{\circ}$ (see also Bao et al. 1997). Here we have assumed that the spot has an intrinsic linear polarization of $60 \%$ (Broderick \& Loeb 2006; Falcke et al. 2000; Goldston et al. 2005). We chose the E-Vector to be perpendicular to the equatorial plane as might be expected for certain magnetic field configurations (see comments above). In addition to the intrinsic polarization we include a $3 \%$ foreground polarization along $30^{\circ}$ (see references above). The combined degree of polarization due the disk, spot and the foreground yield a degree of polarization of up to $20 \%$ and polarization angles that range between $38^{\circ}$ and $83^{\circ}$, which matches what is inferred from the observations.

As can be seen in Fig. 10 our model represents the observed light curve reasonably well within the measurement uncertainties. Regarding the fact that we neglected possible tilts and warps of the accretion disk (e.g. Bardeen \& Petterson 1975; Lubow et al. 2002), magnetic field effects and used a highly idealized and therefore somewhat unphysical intrinsic polarization model, the detailed discrepancy of the polarization angle is not surprising.

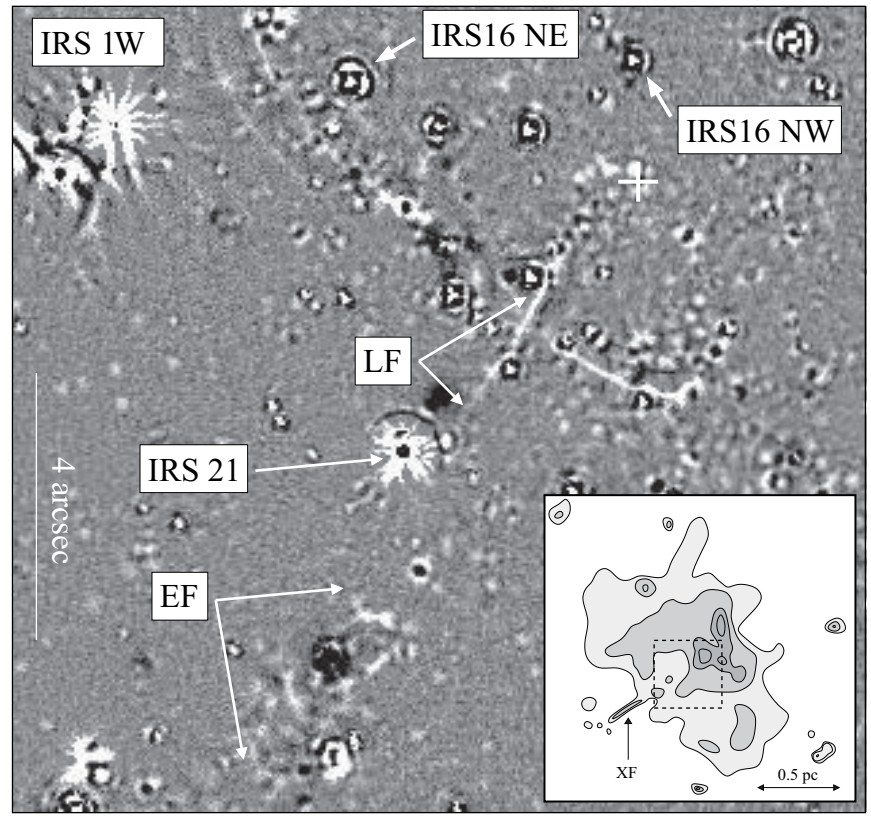

Fig. 9. High pass filtered $L^{\prime}$-band image (centered at $3.8 \mu \mathrm{m}$ wavelength) in which the majority of the extended and stellar flux has been subtracted using the StarFinder code (Diolaiti et al. 2000), i.e. the shown image is a StarFinder residual image. Some residuals are left at the positions of bright stars, some of which have been labeled. The position of SgrA* is marked by the red cross in the north-western quadrant of the map. In addition to a few sharp edged structures associated with the northern arm dust emission of the mini-spiral one can see an linear feature (LF) and an extended structure (EF) both pointing approximately into the direction of SgrA* (see also Fig. 8). The inset shown in the lower right corner is a sketch of the grey scale image shown in Morris et al. (2004). The X-ray feature discussed in the text is labeled as XF. The NIR filament LF and the X-ray filament XF are not colinear. The dashed rectangular line markes the position and extent of the high pass filtered $L^{\prime}$-band image shown here.

\section{Summary and conclusions}

New high angular resolution NIR observations have shown that the flare emission from SgrA* is highly polarized and provide a direct proof of the non-thermal nature of the radiation. At two observing epochs in July 2005 and June 2004 the mean position angle of the $\boldsymbol{E}$-vector was at about $60^{\circ}$. The data can be interpreted in a jet and orbiting disk model. We have discussed possible features that may be indicative for a collimated out-flow or jet from SgrA*. We also find new evidence for a possible quasiperiodicity of $20 \pm 3 \mathrm{~min}$. The new data strengthens the case for a $\mathrm{MBH}$ rather than for alternative explanations of the high mass concentration (see discussion in Appendix B). In the model of compact $\left(\lesssim R_{\mathrm{S}}\right)$ highly polarized hot spots orbiting SgrA* in a temporary disk (see also Genzel et al. 2003a) at a radius of a few times the event horizon (Dovciak et al. 2004; Broderick \& Loeb 2006) this period would be consistent with a MBH spin parameter of $a=0.5$. This supports the presence of a Kerr MBH that rotates at about half its maximum spin and the position angle of the polarized emission may indicate its projected orientation on the sky.

Acknowledgements. This work was supported in part by the Deutsche Forschungsgemeinschaft (DFG) via grant SFB 494. We are grateful to all members of the NAOS/CONICA and the ESO PARANAL team, especially N. Ageorges for support in setting up the polarization measurements. We thank M. Dovciak, A.E. Broderick, and A. Loeb for very helpful discussions and 

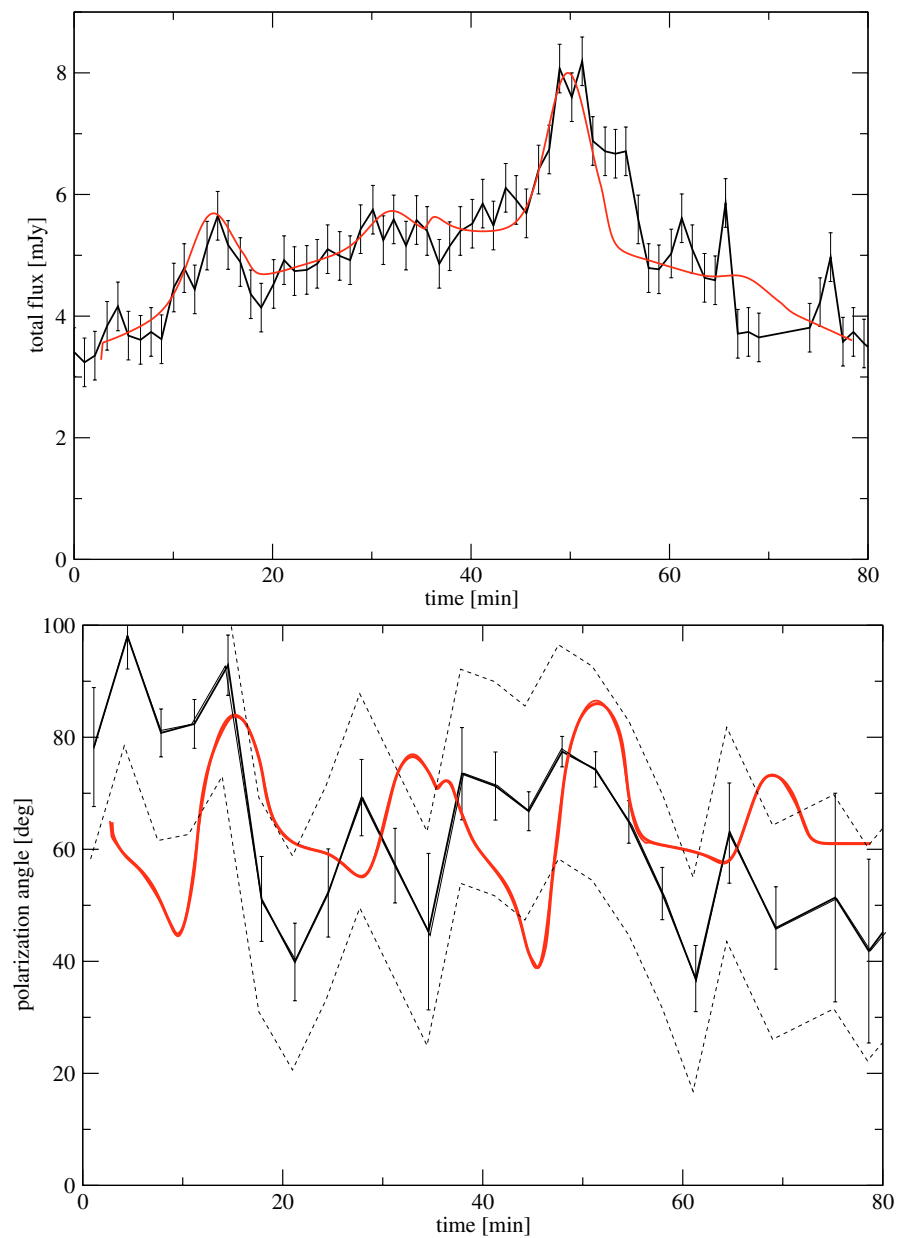

Fig. 10. A comparison between an orbiting spot model (red lines) for July 2005 and the measured total flux density (top) and polarization angle (bottom). As a demonstration of the consistency between the model and the data we have chosen a solution that gives a satisfactory representation of the total flux density. For the chosen inclination of $55^{\circ}$ the position angle of the $\boldsymbol{E}$-vector resulting from the model and interstellar polarization does not wrap and agrees with the measurements to within \pm 3 times the mean $1 \sigma$ uncertainties of the measurements - as indicated by the two thin dashed lines. This solution has to be compared to senarii with different inclinations as shown in the model results in Fig. 7.

especially M. Dovciak for valuable conversations and for providing the code to us. K. Muzic provided the high pass filtered $L^{\prime}$-band image.

\section{Appendix A: Characterization of the flux density variations}

The analysis of variability data by Mauerhan et al. (2005) for SgrA* at $3 \mathrm{~mm}$ wavelength and by (Benlloch et al. 2001) for $\mathrm{X}$-ray data of Mrk 766 have shown that in these cases the flux density variations can well be described by a red-noise power spectrum of the form $P(f) \sim f^{-\beta}$. In order to characterize the variations in our (densely and almost exclusively regularly sampled) polarized NIR flux density data we calculated periodograms using the IDL LNP_TEST function that computes Lomb Normalized Periodograms and is based on the routine "fasper" in Sect. 13.8 of Numerical Recipes in C: The art of Scientific Computing (Cambridge University Press).

To take into account the swing in polarization angle the periodograms were first obtained for data taken at the different individual polarization angles and then averaged. The $1 \sigma$ error bars

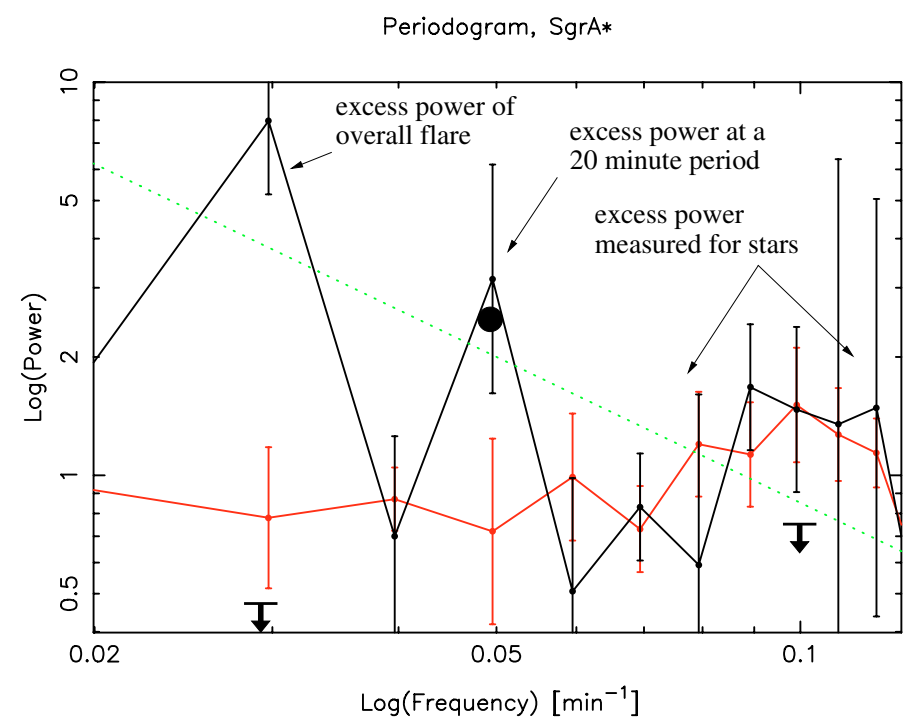

Fig. 11. Top right: double logarithmic periodogram of the July 2005 polarized flux density data from $\mathrm{SgrA}^{*}$ at individual polarization angles. The data for SgrA* is shown in black and the mean and standard deviation calculated from periodograms of 7 stars within 1.5 arcsec radius of SgrA* are shown in red. The green dashed line corresponds to a fit of the data by a power spectrum of the form $P(f) \sim f^{-\beta}$ with an exponent $\beta=1.2 \pm 0.7(1 \sigma)$. Hence our July 2005 NIR data are consistent with a flat noise distribution $(\beta=0)$ and do not provide an indication for a significant low frequency red-noise excess. The value and upper limits printed in bold face are explained in the text.

are derived as the standard deviation of the results for the different polarization angles. The excess power is better visible for angles with high polarized flux. Therefore the $1 \sigma$ error bars are upper limits for the uncertainties of the power values. The result for $\mathrm{SgrA}^{*}$ is shown as a black line in Fig. 11. In Fig. 6 we also show in red the mean and standard deviation calculated from periodograms of 7 stars within 1.5 arcsec radius of SgrA*.

The SgrA* data can be fitted by a power spectrum with an exponent $\beta=1.2$ having a $1 \sigma$ uncertainty of 0.7 . Hence our July 2005 NIR data are consistent with a flat noise distribution $(\beta=0)$ and do not provide an indication for a significant low frequency red-noise excess. The case for red-noise is weakened even further by the fact that the power peaks at frequencies around 0.04 and $0.1 \mathrm{~min}^{-1}$ ( 25 and 10 min periods) can be attributed to the overall unpolarized part of the flare and a noise pattern that is also contained in the stellar periodograms, respectively. The excess power around $0.1 \mathrm{~min}^{-1}$ (10 min period) corresponds to the frequency of dithering and therefore most likely derives from residual uncertainties of the calibration and data processing. Both SgrA* and the stars show a similarly shaped excess between frequencies of 0.2 to $0.07 \mathrm{~min}^{-1}$ (5 to $15 \mathrm{~min}$ periods) and in Fig. 11 the two periodograms have been normalized to the excess power in this range. There is a remaining relative scatter between the two curves of $1 \sigma \sim 0.25$. The resulting $3 \sigma$ limit for intrinsic (combined polarized and unpolarized) power of SgrA* in this frequency range is shown as a bold face upper limit.

The excess power contained in the $\mathrm{SgrA}^{*}$ data at a frequency of $0.05 \mathrm{~min}^{-1}$ (a $20 \mathrm{~min}$ period) is not present in the stellar data. We identify this excess with the power contained in the polarized portion of the SgrA* flare. In Fig. 11 we plot in bold face the power contained in the light curve of $\mathrm{SgrA}^{*}$ at this frequency corrected for the power contribution also present in the stellar light curves. 
Using the fact that the relative powers relate as the integrals under the corresponing portions of the light curves we can correct for the power contained in the unpolarized part of the overall flare emission at a frequency of $0.05 \mathrm{~min}^{-1}$. We then obtain an upper limit for the polarized power at that frequency which is shown in bold face in Fig. 11. This number is an upper limit since the power contained in the polarized portion of the light curve clearly occurs at higher frequencies.

Since the SgrA* periodogram does not contain a dominant contribution of red-noise the significance standards of Horne \& Baliunas (1986) apply and we regard the excess power at a frequency of $0.05 \mathrm{~min}^{-1}$ ( $20 \mathrm{~min}$ period) as a significant indication for a quasi-periodicty. The physical interpretation of this excess is discussed in the paper Sects. 3 and 7.

\section{Appendix B: Alternative models}

Explaining SgrA* with alternative solutions for a MBH becomes increasingly difficult. Stellar orbits near SgrA* make a universal Fermion ball solution for compact galactic nuclei highly unlikely (Schödel et al. 2002; Maoz 1998; see also Melia \& Falcke 2001; Yuan et al. 2004). The good agreement between the measured polarized flare structure and the theoretical predictions (Broderick \& Loeb 2006; Eisenhauer et al. 2005; Ghez et al. 2004; Gillessen 2006) as well as the indication of a quasiperiodicity in the data severely challenge the alternative explanation of the central mass as a massive boson star (Torres et al. 2000; Mielke \& Schunck 2000; Lu \& Torres 2003, and references therein). If an ad hoc weak repulsive force between a hypothetical brand of bosons is introduced, it appears to be possible to form massive, compact objects with sizes of a few $R_{\mathrm{S}}$ that are supposedly supported by the Heisenberg uncertainty principle. However, it is a delicate process to form a boson star and preventing it from collapsing to a MBH despite of further accretion of matter, a non spherically symmetric arrangement of forces as in the case of a jet or matter being in orbit around the center but well within the boson star. Such a massive boson star scenario could already be excluded for the nucleus of MCG-6-30-15 (Lu $\&$ Torres 2003). In the case of a stationary boson star the orbital velocity close to the $\sim 3 R_{\mathrm{S}}$ radius LSO is already $\sim 3$ times lower than that of a Schwarzschild MBH (Lu \& Torres 2003) and relativistic effects are severely diminished and further reduced at even smaller radii. If the indicated quasi-periodicity is due to orbital motion then a stationary boson star can be excluded as an alternative solution for $\mathrm{SgrA}^{*}$, since in this case one expects the orbital periods to be larger.

\section{References}

Aitken, D. K., Greaves, J., Chrysostomou, A., et al. 2000, ApJ, 534, L173 Baganoff, F. K., Bautz, M. W., Brandt, W. N., et al. 2001, Nature, 413, 45 Baganoff, F. K., Maeda, Y., Morris, M., et al. 2003, ApJ, 591, 891 Balbus, S. A. 2003, ARA\&A, 41, 555

Balbus, S. A., \& Hawley, F. H. 1991, ApJ, 376, 214

Bao, G., Hadrava, P., Wiita, P. J., et al. 1997, ApJ, 487, 142

Bardeen, J. M., \& Petterson, J. A. 1975, ApJ, 195, L65

Belanger, G., Goldwurm, A., Melia, F., et al. 2005, ApJ, 635, 1095

Benlloch, S., Wilms, J., Edelson, R., Yaqoob, T., \& Straubert, R. 2001, ApJ, 562, L124

Bower, G. C., Wright, M. C. H., Backer, D. C., \& Falcke, H. 1999a, ApJ, 527, 851

Bower, G. C., Falcke, H., \& Backer, D. C. 1999b, ApJ, 523, L29

Bower, G. C., Backer, D. C., Zhao, J.-H., Goss, M., \& Falcke, H. 1999c, ApJ, 521,582
Bower, G. C., Wright, M. C. H., Falcke, H., \& Backer, D. C. 2001, ApJ, 555, L103

Bower, G. C., Falcke, H., \& Mellon, R. R. 2002a, ApJ, 578, L103

Bower, G. C., Falcke, H., Sault, R. J., \& Backer, D. C. 2002b, ApJ, 571, 843

Bower, G. C., Wright, M. C. H., Falcke, H., \& Backer, D. C. 2003, ApJ, 588, 331

Bower, G. C., Falcke, H., Herrnstein, R. M., et al. 2004, Science, 304, 704 Broderick, A. E., \& Loeb, A. 2006, MNRAS, 367, 905

Clénet, Y., Rouan, D., Gendron, E., et al. 2004, A\&A, 417, L15

Diolaiti, E., Bendinelli, O., Bonaccini, D., et al. 2000, A\&AS, 147, 335

Dovciak, M., Karas, V., \& Yaqoob, T. 2004, ApJS, 153, 205

Eckart, A., Genzel, R., Hofmann, R., et al. 1995, ApJ, 445, L23

Eckart, A., Genzel, R., Ott, T., \& Schödel, R. 2002, MNRAS, 331, 917

Eckart, A., Baganoff, F. K., Morris, M., et al. 2004, A\&A, 427, 1

Eckart, A., Schödel, R., \& Straubmeier, C. 2005, The black hole at the center of the Milky Way (London: Imperial College Press)

Eckart, A., Baganoff, F. K., Schödel, R., et al. 2006, A\&A, 450, 535

Eisenhauer, F., Schödel, R., Genzel, R., et al. 2003, ApJ, 597, L121

Eisenhauer, F., Genzel, R., Alexander, T., et al. 2005, ApJ, 628, 246

Falcke, H., Melia, F., \& Agol, E. 2000, ApJ, 528, L13

Fender, R. 2003, Ap\&SS, 288, 79

Fender, R., Rayner, D., Norris, R., Sault, R. J., \& Pooley, G. 2000, ApJ, 530, L29

Gabuzda, D. C., Pushkarev, A. B., \& Cawthorne, T. V. 2000, MNRAS, 319, 1109

Genzel, R., Schödel, R., Ott, T., et al. 2003a, Nature, 425, 934

Genzel, R., Schödel, R., Ott, T., et al. 2003b, ApJ, 594, 812

Ghez, A. M., Wright, S. A., Matthews, K., et al. 2004, ApJ, 601, L159

Ghez, A. M., Salim, S., Hornstein, S. D., et al. 2005, ApJ, 620, 744

Gillessen, S., Eisenhauer, F., Quataert, E., et al. 2006, ApJ, 640, L163

Goldston, J. E., Quataert, E., \& Igumenshchev, I. V. 2005, ApJ, 621, 785

Gomez, J. L., Alberdi, A., \& Marcaide, J. M. 1994, A\&A, 284, 51

Herrnstein, R. M., Zhao, J.-H., Bower, G. C., \& Goss, W. M. 2004, AJ, 127, 3399

Horne, J. H., \& Baliunas, S. L. 1986, ApJ, 302, 757

Knacke, R. F., \& Capps, R. W. 1977, ApJ, 216, 271

LaRosa, T. N., Nord, M. E., Lazio, T. J. W., \& Kassim, N. E. 2004, ApJ, 607, 302

Lebofsky, M. J., Rieke, G. H., Deshpande, M. R., \& Kemp, J. C. 1982, ApJ, 263, 672

Levin, Y., \& Beloborodov, A. M. 2003, ApJ, 590, L33

Lu, Y., \& Torres, D. F. 2003, Int. J. Mod. Phys. D, 12, 63

Lubow, S. H., Ogilvie, G. I., \& Pringle, J. E. 2002, MNRAS, 337, 706

Lutz, D., Krabbe, A., \& Genzel, R. 1993, ApJ, 418, 244

Mauerhan, J. C., Morris, M., Walter, F., \& Baganoff, F. K. 2005, ApJ, 623, 25

Markoff, S., Falcke, H., Yuan, F., \& Biermann, P. L. 2001, A\&A, 379, L13

Maoz, E. 1998, ApJ, 494, L181

Marrone, D. P., Moran, J. M., Zhao, J.-H., \& Rao, R. 2006, ApJ, 640, 308

Melia, F., \& Falcke, H. 2001, ARA\&A, 39, 309

Melia, F., Coker, R. F., \& Yusef-Zadeh, F. 1996, ApJ, 460, L33

Mielke, E., \& Schunck, F. 2000, Nucl. Phys. B, 594, 1985

Morris, M., Howard, C., Muno, M., et al. 2004, in The Dense Interstellar Medium in Galaxies, Proc. of the 4th Cologne-Bonn-Zermatt Symposium, Zermatt, Switzerland, 22-26 September 2003, ed. S. Pfalzner, C. Kramer, C. Staubmeier, \& A. Heithausen, Springer proceedings in physics (Berlin, Heidelberg: Springer), 91, 281

Morris, M. 1996, Nature, 383, 389

Morris, M., \& Serabyn, E. 1996, ARA\&A, 34, 645

Nord, M. E., Lazio, T. J. W., Kassim, N. E., et al. 2004, AJ, 128, 1646

Ott, T., Eckart, A., Genzel, R., et al. 1999, ApJ, 523, 248

Paumard, T., Maillard, J.-P., \& Morris, M. 2004, A\&A, 426, 81

Pollack, L. K., Taylor, G. B., \& Zavala, R. T. 2003, ApJ, 589, 733

Pushkarev, A. B., Gabuzda, D. C., Vetukhnovskaya, Yu. N., \& Yakimov, V. E. 2005, MNRAS, 356, 859

Rusk, R. 1988, in The Impact of VLBI on Astrophysics and Geophysics, Proc. of the 129th IAU Symp., Cambridge, MA, May 10-15, 1987, ed. M. J. Reid, \& J. M. Moran (Dordrecht: Kluwer Academic Publishers), 161

Schödel, R., Ott, T., Genzel, R., et al. 2002, Nature, 419, 694

Schödel, R., Genzel, R., Ott, T., et al. 2003, ApJ, 596, 1015

Shakura, N. I., \& Sunyaev, R. A. 1973, A\&A, 24, 337

Torres, D. F., Capozziello, S., \& Lambiase, G. 2000, Phys. Rev. D, 62, 104012 Vollmer \& Duschl 2000, New Astron., 4, 581

Yuan, Y.-F., Narayan, R., \& Rees, M. J. 2004, ApJ, 606, 1112

Yuan, F., Markoff, S., \& Falcke, H. 2002, A\&A, 854, 854

Yuan, F., Quataert, E., \& Narayan, R. 2004, ApJ, 606, 894

Yusef-Zadeh, F., Roberts, D. A., \& Biretta, J. 1998, ApJ, 499, L159

Yusef-Zadeh, F., et al. 2006, ApJ, in press [arXiv: astro-ph/0510787] 